\title{
Modelo de Lotka - Volterra en la biomatemática Solución de sistema depredador-presa
}

\author{
Lotka - Volterra model in biomatematics \\ Predator-prey system solution
}

${ }^{1}$ Luis Andrés Amaya Cedrón

ORCID: 0000-0002-1172-8422

\section{RESUMEN}

En la investigación, el modelo presa-depredador, también conocido como el modelo Lotka-Volterra, ha sido un punto de partida para el desarrollo de nuevas técnicas y teorías matemáticas. El modelo presadepredador se ocupa de la interacción entre dos especies, donde una de ellas (presa) tiene abundante comida y la segunda especie (depredador) tiene suministro de alimentos exclusivamente a la población de presas. Donde se supone también que, durante el proceso, en un intervalo de tiempo t, el medio no debería cambiar favoreciendo a ninguna de las especies y que cualquier adaptación genética es lo suficientemente lenta (Figueiredo, 2014). En este trabajo haremos el análisis de problema clásico de Presa-depredador.

Palabras claves: modelo Lotka-Volterra, pesa-depredador.

\begin{abstract}
In research, the prey-predator model, also known as the Lotka-Volterra model, has been a starting point for the development of new mathematical techniques and theories. The prey-predator model deals with the interaction between two species, where one of them (prey) has abundant food and the second species (predator) has food supply exclusively to the prey population. Where it is also assumed that, during the process, in a time interval $t$, the environment should not change favoring any of the species and that any genetic adaptation is slow enough (Figueirero, 2014). In this work we will do the classic Prey-predator problem analysis.
\end{abstract}

Keywords: Lotka-Volterra model, prey-predator.

\footnotetext{
${ }^{1}$ Universidad Nacional Jorge Basadre Grohmann. Tacna, Perú. E-mail: lamyac@unjbg.edu.pe 


\section{INTRODUCCIÓN}

Entre los modelos de interacción entre especies, estudiaremos el modelo clásico de depredador que, por su simplicidad y belleza, cautivó a un gran número de investigadores que comenzaron a usarlo como paradigma de sus modelos modificados. El modelo presa-depredador, también conocido como el modelo Lotka-Volterra, se ocupa de la interacción entre dos especies, donde una de ellas (presa) tiene abundante comida, y la segunda especie (depredador) tiene suministro de alimentos exclusivamente a la población de presas (Figueirero, 2014).

Supongamos también que, durante el proceso, en un intervalo de tiempo t, el medio no debería cambiar favoreciendo a ninguna de las especies y que cualquier adaptación genética es lo suficientemente lenta.

Las variaciones están dadas por las siguientes ecuaciones:

$$
\left\{\begin{array}{l}
\left(\begin{array}{c}
\text { variación } \\
\text { de número de } \\
\text { presas }
\end{array}\right)=\left(\begin{array}{l}
\text { aumento } \\
\text { natural }
\end{array}\right)-\left(\begin{array}{l}
\text { destrucción por } \\
\text { depredadores }
\end{array}\right) \\
\left(\begin{array}{c}
\text { variación } \\
\text { de número de } \\
\text { depredadores }
\end{array}\right)=-\left(\begin{array}{c}
\text { muerte en } \\
\text { ausencia } \\
\text { de presa }
\end{array}\right)+\left(\begin{array}{l}
\text { aumento } \\
\text { causadopor } \\
\text { alimentación } \\
\text { disponible }
\end{array}\right)
\end{array}\right.
$$

$$
\begin{aligned}
& x=x(t): \text { la densidad poblacional de las presas, } \mathrm{y} \\
& y=y(t): \text { la densidad de población de depredadores de presas, } \\
& \text { en cada momento } t \text {. }
\end{aligned}
$$

\section{MATERIAL Y MÉTODOS}

En este trabajo se utilizó material bibliográfico sobre de ecuaciones diferenciales parciales de primer orden, además, se usó el software de cálculo simbólico Matlab.

Respecto al método utilizado, se realizó el estudio bibliográfico acerca de sistemas de ecuaciones diferenciales parciales de primer orden y en biología sobre poblaciones, dinámica de poblaciones, curva de crecimiento.

\section{RESULTADOS Y DISCUSIÓN}

\section{Modelo de Lotka-Volterra}

En pocas palabras, el modelo de Lotka-Volterra supone que las presas crecen exponencialmente en ausencia de depredadores (modelo de Malthus) y que la tasa de mortalidad de depredadores en ausencia de presa es proporcional a su población $\boldsymbol{y}(\boldsymbol{t})$ en cada momento $\boldsymbol{t}$ (muerte por falta de alimentos) (Puchuri, 2018). La tasa de natalidad del depredador depende exclusivamente de este modelo en la cantidad de presas devoradas en cada encuentro. 
Si modelamos los posibles encuentros por el término bilineal $\boldsymbol{x} \boldsymbol{y}$, entonces el sistema presadepredador, simplificada por las imposiciones anteriores, viene dada según Puchuri (2018) por:

$$
\left\{\begin{array}{l}
\frac{d x}{d t}=a x-b x y \\
\frac{d y}{d t}=-c y+d x y
\end{array}\right.
$$

Recordemos que en el modelo clásico de Lotka-Volterra se considera un hábitat en donde coexisten dos especies que interaccionan entre ellas. La especie $x(t)$ se le llamará presa y tiene una fuente de alimentación por la que no compite; la otra especie $y(t)$, a la que llamaremos depredador, tiene a $x(t)$ en su dieta. De esta forma, $x(t)$ representa el número de presas en el instante $t$, mientras que $y(t)$ indica la cantidad de depredadores en ese mismo momento (Puchuri, 2018).

Hipótesis más simples del problema depredador presa:

$$
\begin{aligned}
& \frac{d x}{d t}=a x(t): \text { Las presas, en ausencia de cualquier depredador. } \\
& \frac{d x}{d t}=a x(t)-b x(t) y(t): \text { Si hay presencia de depredador. } \\
& \frac{d y}{d t}=-c y(t): \text { Si no hay presas } \\
& \frac{d y}{d t}=-c x(t)-d x(t) y(t):
\end{aligned}
$$

La contribución de las presas a la tasa de crecimiento relativa de los depredadores.

Donde los parámetros $a, b, c$ y $d$ son constantes positivas (Puchuri, 2018).

\section{Análisis del modelo}

Buscamos, por tanto, funciones $(x(t), y(t))$ que sean solución del sistema de la ecuación anterior (1). Obviamente, esta solución dependerá de la cantidad inicial presente de depredadores y presa, es decir, de $(x(0), y(0))$. No obstante, estudiando la ecuación, podemos obtener ya algunas soluciones particulares de esta.

1. $x(t)=y(t)=0, t \in \mathrm{R}$ : solución trivial

2. $y(t)=y(0) e^{-c t}, \quad t \in R$ : exponencial decreciente, si población de presas se reduce a cero, $x(t)$ $=0$

3. Si no hay depredadores, $y(t)=0$, observamos que la evolución de la población de presas se ajustaría a un modelo malthusiano de crecimiento. $x(t)=x(0) e^{a t}, \quad y(t)=0, t \in R$.

\section{Puntos de equilibrio}

Los puntos de equilibrio en este sistema son los que se dan cuando la variación de la densidad de la población es nula.

Según José López (2012), para calcular los puntos de equilibrios se resuelve el siguiente sistema no lineal 


$$
\left\{\begin{array}{l}
a x-b x y=0 \\
-c y+d x y=0
\end{array}\right.
$$

Por tanto, los únicos puntos de equilibrio son los $Q=(0,0)$ y el $P=\left(\frac{c}{d}, \frac{a}{b}\right)$. Analizando el punto $Q=(0,0)$ se tiene que:

Cuya matriz asociada es

$$
J(x, y)=\left[\begin{array}{cc}
a-b y & -b x \\
d y & -c+d x
\end{array}\right]
$$

$$
J(0,0)=\left[\begin{array}{cc}
a & 0 \\
0 & -c
\end{array}\right]
$$

Según la matriz, se tienen $\operatorname{los}$ auto valores $\lambda_{1}=a>0 \mathrm{y} \lambda_{2}=-c<0$, por lo que el punto de equilibrio es un nodo inestable, en otras palabras en un punto silla.

Analizamos el punto $P=\left(\frac{c}{d}, \frac{a}{b}\right)$; para ello, se hace un cambio de variable $\left\{u=x-\frac{c}{d} ; \quad v=y-\frac{a}{b}\right.$ se obtienen las ecuaciones

$$
\left\{\begin{array}{l}
\frac{d u}{d t}=-b \frac{c}{d} v-b u v \\
\frac{d v}{d t}=d \frac{a}{b}+d u v
\end{array}\right.
$$

Donde ahora se tiene que $(0,0)$ es un punto de equilibrio y se tiene

$$
\begin{aligned}
& J(x, y)=\left[\begin{array}{cc}
-b v & -b \frac{c}{d}-b u \\
d \frac{a}{b}+d v & d u
\end{array}\right] \Rightarrow \\
& J(0,0)=\left[\begin{array}{cc}
0 & -b \frac{c}{d} \\
d \frac{a}{b} & 0
\end{array}\right]
\end{aligned}
$$

Los autovalores de esta matriz se obtienen al resolver el polinomio $\lambda^{2}+a c=0$ y resulta $\lambda= \pm \sqrt{a c} i$, lo que muestra que $P=\left(\frac{c}{d}, \frac{a}{b}\right)$ es un centro y sugiere que las soluciones girarán en torno a él dando lugar, en este caso, a soluciones periódicas de las poblaciones. Las soluciones son ahora. 


$$
\begin{gathered}
\left\{\begin{array}{l}
x(t)=c_{1} \cos \sqrt{a c} t+c_{2} \operatorname{sen} \sqrt{a c} t \\
y(t)=c_{1}(A) \operatorname{sen} \sqrt{a c} t+c_{2}(-A) \cos \sqrt{a c} t
\end{array}\right. \\
\text { con } A=\frac{d}{c} \sqrt{\frac{a}{b}}
\end{gathered}
$$

Es decir, como tenemos dos auto valores imaginarios conjugados, el punto es punto de centro. $\mathrm{O}$ sea, las curvas de las soluciones son elipses centradas en el punto de equilibrio.

\section{Solución Sistemas depredador-presa}

"Modelos que tienen en cuenta la interacción de dos especies en el mismo hábitat"

\section{Ecuación general}

Un sistema de dos ecuaciones diferenciales que incorpora estos supuestos es:

$$
\begin{aligned}
& \frac{d x}{d t}=a x-b x \cdot y \\
& \frac{d y}{d t}=-c y+d x \cdot y
\end{aligned}
$$

\section{Ejemplo 1.}

Suponga que las poblaciones de conejos y lobos se describen mediante las ecuaciones de LotkaVolterra con: $a=0.08, \mathrm{~b}=0.001, \mathrm{c}=0.02, \mathrm{~d}=0.00002$

El tiempo $t$ se mide en meses.

a) Encuentre las soluciones constantes (llamadas soluciones de equilibrio) e interprete la respuesta.

Con los valores dados de $a, b, c$ y $d$, las ecuaciones de Lotka-Volterra se convierten en:

$$
\left\{\begin{array}{l}
\frac{d x}{d t}=0,08 x-0,001 x \cdot y \\
\frac{d y}{d t}=-0,02 y+0,00002 x \cdot y
\end{array}\right.
$$

Donde $a=0.08 ; b=0.001 ; c=0.02$ y $d=0.00002$.

Tanto $\boldsymbol{x}$ como $\boldsymbol{y}$ serán constantes si ambas derivadas son 0 . Es decir, 


$$
\begin{aligned}
& \left\{\begin{array}{l}
\frac{d x}{d t}=0,08 x-0,001 x \cdot y=0 \\
\frac{d y}{d t}=-0,02 y+0,00002 x y=0
\end{array}\right. \\
& \Rightarrow\left\{\begin{array}{l}
x(0,08-0,001 y)=0 \\
y(-0,02+0,00002 x)=0
\end{array}\right.
\end{aligned}
$$

Una solución viene dada por: $(x, y)=(0,0)$. Esto tiene sentido.

Si no hay conejos ni lobos, las poblaciones ciertamente no van a aumentar.

La otra solución constante es:

$$
\begin{aligned}
& \left\{\begin{array}{l}
x(0,08-0,001 y)=0 \\
y(-0,02+0,00002 x)=0
\end{array}\right. \\
& \Rightarrow\left\{\begin{array} { l } 
{ ( 0 , 0 8 - 0 , 0 0 1 y ) = 0 } \\
{ ( - 0 , 0 2 + 0 , 0 0 0 0 2 x ) = 0 }
\end{array} \Rightarrow \left\{\begin{array}{c}
x=1000 \\
y=80
\end{array}\right.\right.
\end{aligned}
$$

Entonces, las poblaciones de equilibrio consisten en 80 lobos y 1000 conejos.

b) Usamos el sistema de Ecs. Dif para encontrar una expresión para $\frac{d y}{d x}$

Usamos la regla de la cadena para eliminar t: $\frac{d y}{d t}=\frac{d y}{d x} \frac{d x}{d t}$, por lo tanto,

$$
\frac{d y}{d x}=\frac{\frac{d y}{d t}}{\frac{d x}{d t}}=\frac{-0.02 y+0.00002 x y}{0.08 x-0.001 x y}
$$

c) Dibujamos un campo de dirección para la ecuación diferencial resultante en el plano xy.

$$
\begin{aligned}
& \frac{d y}{d x}=\frac{-0.02 y+0.00002 x y}{0.08 x-0.001 x y} \Rightarrow \\
& \left(\frac{0.08-0.001 y}{y}\right) d y=\left(\frac{-0.02+0.00002 x}{x}\right) d x \\
& \int\left(\frac{0.08-0.001 y}{y}\right) d y=\int\left(\frac{-0.02+0.00002 x}{x}\right) d x \\
& \Rightarrow 0.08 \ln y-0.001 y=-0.02 \ln x+0.00002 x+C \\
& \Rightarrow 0.08 \ln y-0.001 y+0.02 \ln x-0.00002 x=C t e
\end{aligned}
$$


Es decir: Teniendo $\mathrm{a}=0.08 ; \mathrm{b}=0.001 ; \mathrm{c}=0.02 \mathrm{y} \mathrm{d}=0.00002$

Luego, reemplazando en:

$a \ln y-b y=d x-c \ln x+c t e$

$\Rightarrow 0.08 \ln y-0.001 y-0.00002 x+0.02 \ln x=c t e$

Dibujamos el campo de dirección para la ecuación diferencial.

\section{Figura 1}

Campo de dirección

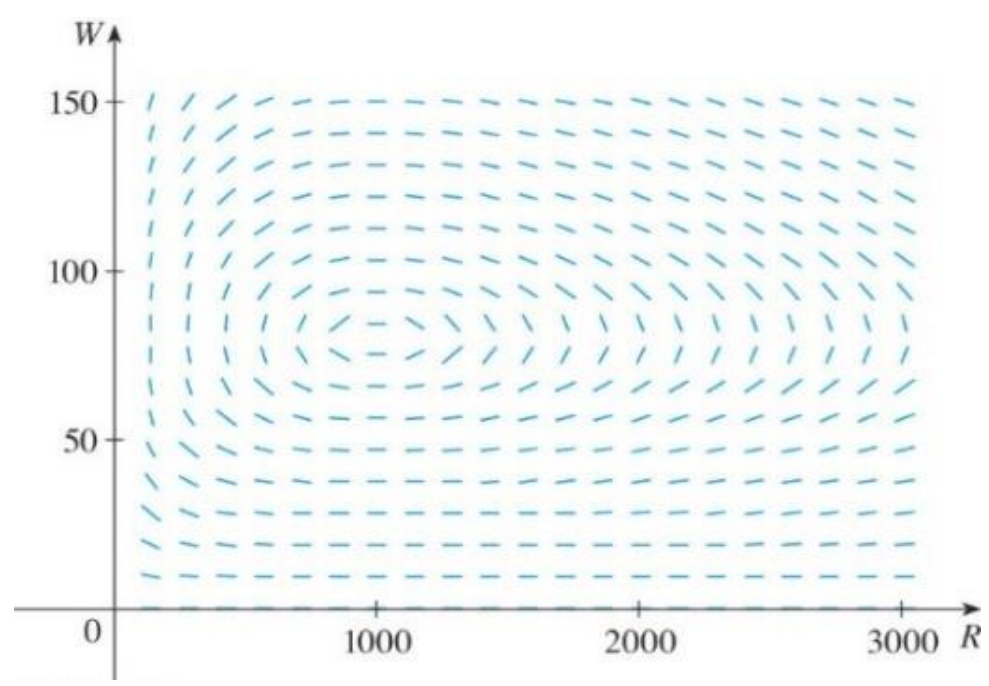

Fuente: Elaboración propia

Luego, usamos el campo para dibujar varias curvas de solución.

c.1) Si reemplazamos el punto (x, y) en la solución, $0.08 \ln y-0.001 y-0.00002 x+0.02 \ln x=$ cte determinamos la cte.

\section{Tabla 1}

Puntos por donde pasa cada curva

\begin{tabular}{ll}
\hline \multicolumn{1}{c}{$(\mathbf{x}, \mathbf{y})$} & \multicolumn{1}{c}{ Cte. } \\
\hline $\mathbf{( 1 0 0 0 , 8 0 )}$ & 0.3837 \\
$\mathbf{( 1 0 0 0 , 1 2 0 )}$ & 0.3812 \\
$(\mathbf{1 0 0 0 , 6 0 )}$ & 0.3857 \\
$\mathbf{( 1 0 0 0 , 4 0 )}$ & 0.3733 \\
\hline
\end{tabular}




\section{Figura 2}

Solución para los diferentes puntos. L.V.

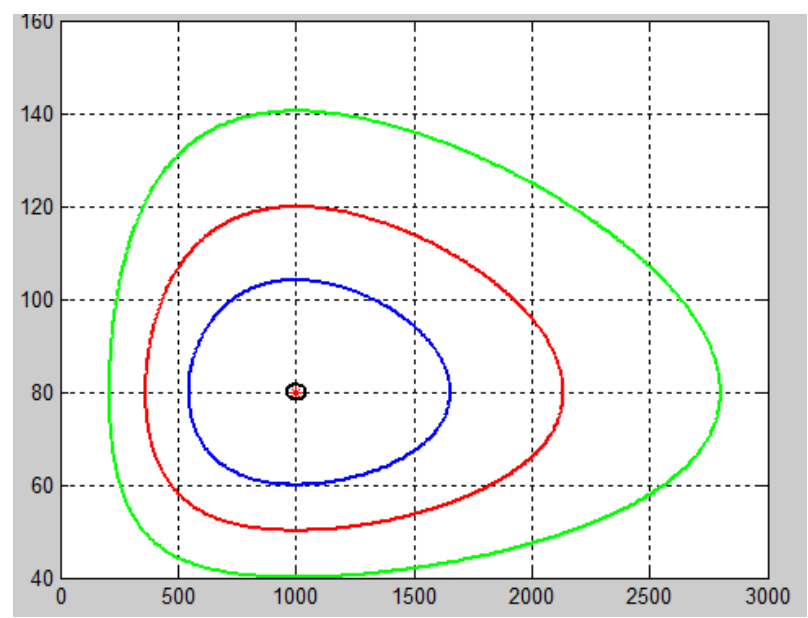

Fuente: Elaboración propia

"Si nos movemos a lo largo de una curva de solución, observamos cómo cambia la relación entre $\boldsymbol{x}(\boldsymbol{t})$ y $\boldsymbol{y}(\boldsymbol{t})$ a medida que pasa el tiempo".

Luego, llamamos a las curvas de solución las trayectorias de fase.

Entonces, una trayectoria de fase es un camino trazado por soluciones $(x, y)$ a medida que pasa el tiempo.

d) Supongamos que, en algún momento, hay 1000 conejos y 40 lobos. Dibujamos la curva de solución correspondiente y la usamos para describir los cambios en ambos niveles de población.

\section{Figura 3}

Trayectoria de fase, analizando $P o, P_{1}, P_{2}, P_{3}$

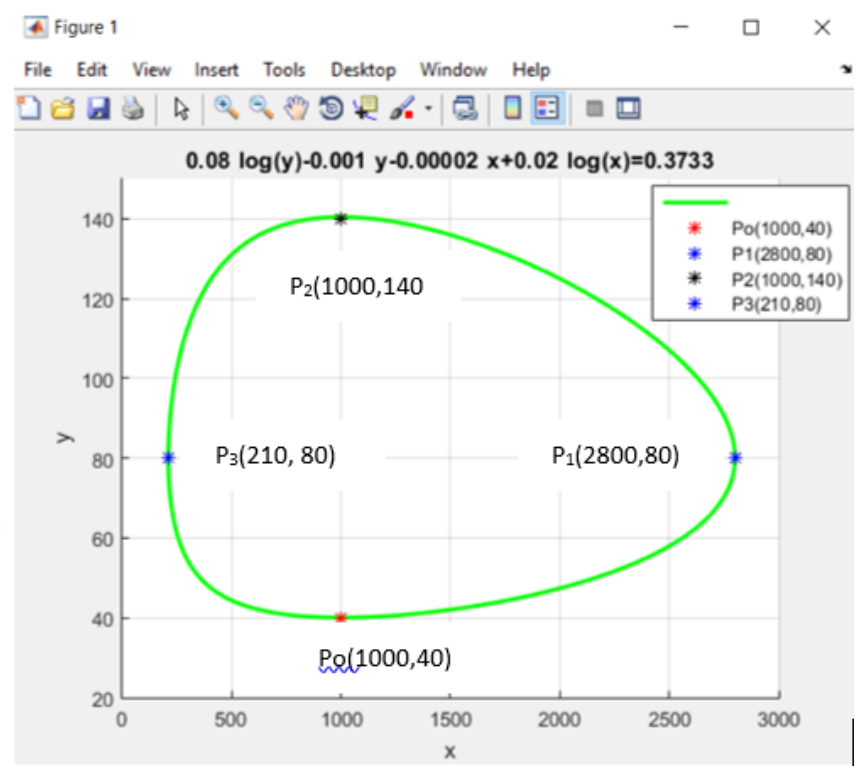

Fuente: Elaboración propia 
Si ponemos $x=1000$ y $y=40$ en la primera ecuación diferencial, obtenemos:

$\frac{d x}{d t}=0.08 x-0.001 x y=0.08(1000)-0.001(1000)(40)=80-40=40$

Como $\frac{d x}{d t}>0$, concluimos que $x$ está aumentando en $\mathrm{P}_{0}$.

Entonces, nos movemos en sentido antihorario alrededor de la trayectoria de la fase. Vemos que, en $\mathrm{P}_{0}$, no hay suficientes lobos para mantener un equilibrio entre las poblaciones. En $\mathrm{P}_{1}$, donde estimamos que $\boldsymbol{x}$ alcanza su población máxima de aproximadamente 2800. Entonces, la población de conejos aumenta. Eso da como resultado más lobos. Finalmente, hay tantos lobos que los conejos tienen dificultades para evitarlos. Por lo tanto, el número de conejos comienza a disminuir y la población de lobos aumentan, Esto está en $\mathrm{P}_{2}$, donde $x=1000$ y $y \approx 140$. Esto significa que, en algún momento posterior, la población de conejos disminuye y lobos comienza a aumentar.

Pero si disminuyen los conejos, entonces disminuyen los lobos $\mathrm{P}_{3}$. Sin embargo, esto beneficia a los conejos. Hasta llegar a los valores iniciales $(x=1000, y=40)$, todo el ciclo comienza de nuevo. Usamos estos resultados para hacer bocetos de $\boldsymbol{x}$ y $\boldsymbol{y}$ como funciones de $\mathrm{t}$.

Es decir, a partir de la descripción en de cómo aumentan y disminuyen las poblaciones de conejos y lobos, podemos dibujar los gráficos de $x(t)$ y $y(t)$.

Tenemos: El origen de coordenadas es $P=\left(\frac{c}{d}, \frac{a}{b}\right)=(1000,80)$

Además $J(0,0)=\left[\begin{array}{cc}0 & -b \frac{c}{d} \\ d \frac{a}{b} & 0\end{array}\right]=\left[\begin{array}{cc}0 & 1 \\ 0,0016 & 0\end{array}\right]$

Hallando los autovalores:

$$
\begin{aligned}
\operatorname{det}(J-\lambda I) & =\left|\left[\begin{array}{cc}
0 & -1 \\
0.0016 & 0
\end{array}\right]-\left[\begin{array}{ll}
\lambda & 0 \\
0 & \lambda
\end{array}\right]\right| \\
& =\left|\left[\begin{array}{cc}
-\lambda & -1 \\
0.0016 & -\lambda
\end{array}\right]\right|=\lambda^{2}+0,0016
\end{aligned}
$$

Igualando a cero: $\lambda= \pm \sqrt{-0,0016}= \pm 0,04 i$

La solución es:

$$
\begin{aligned}
& \left\{\begin{array}{l}
x(t)=c_{1} \cos 0,04 t+c_{2} \operatorname{sen} 0,04 t \\
y(t)=c_{1}(0,0089) \operatorname{sen} 0,04 t+c_{2}(-0,0089) \cos 0,04 t
\end{array}\right. \\
& \text { con } A=\frac{d}{c} \sqrt{\frac{a}{b}}=0,001 * 8,94=0,0089
\end{aligned}
$$




\section{Programa para graficar el comportamiento de conejos $x(t)$ y lobos $y(t)$}

Programa Comportamiento del Número de Presas y Depredador

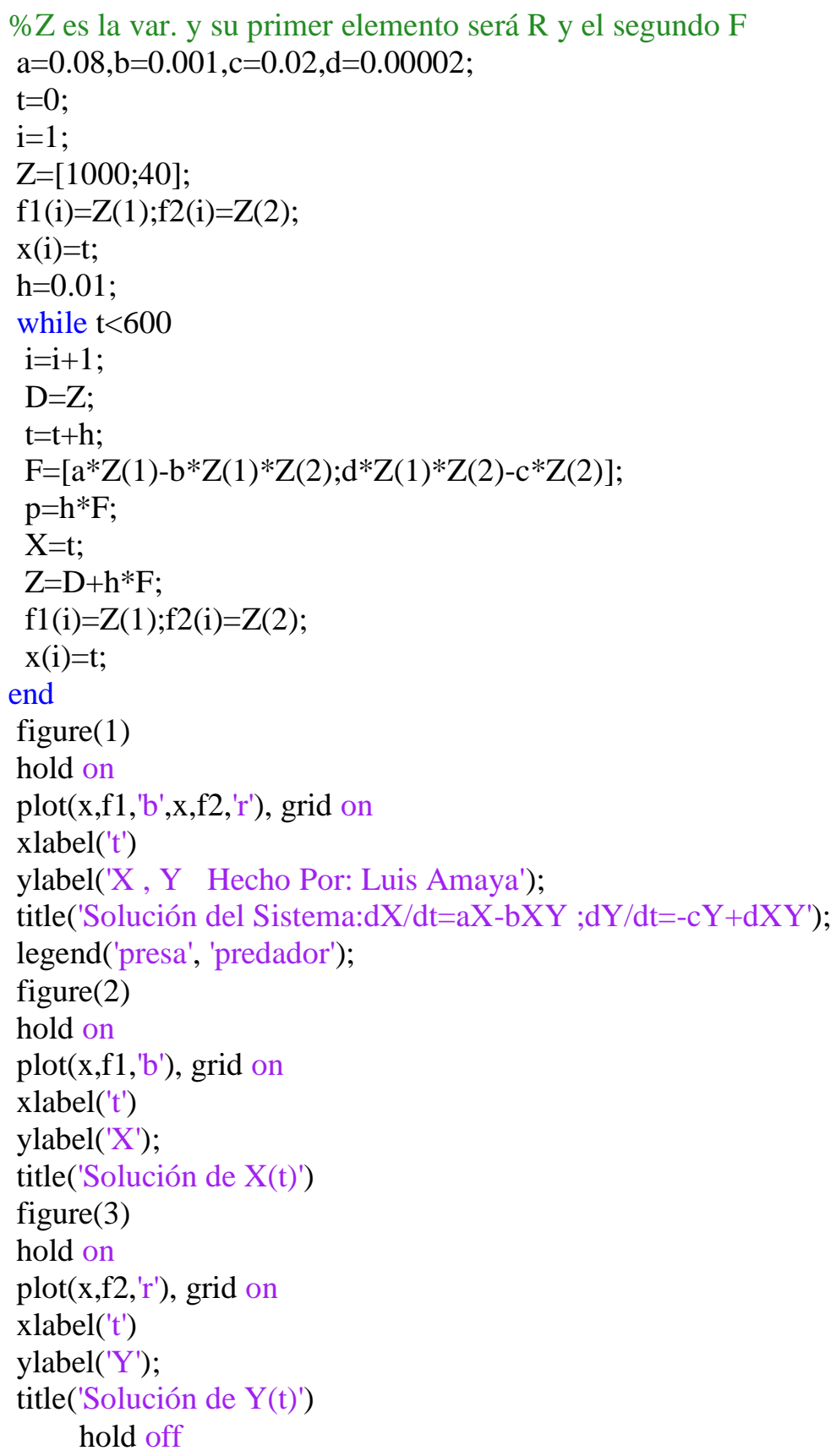


Figura 4

Comportamiento del número de presas y depredador

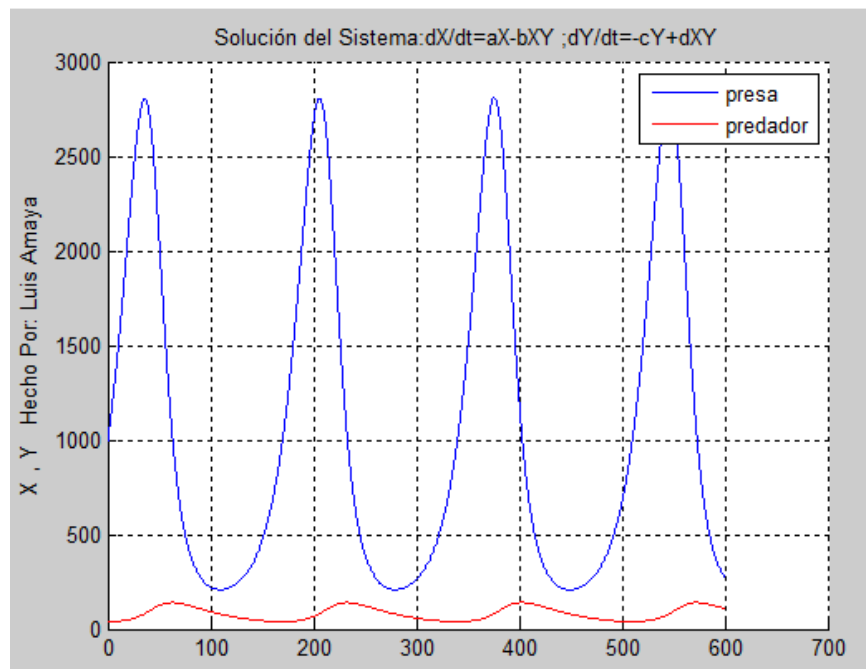

Fuente: Elaboración propia

Figura 5

Comportamiento del número de presas en el tiempo

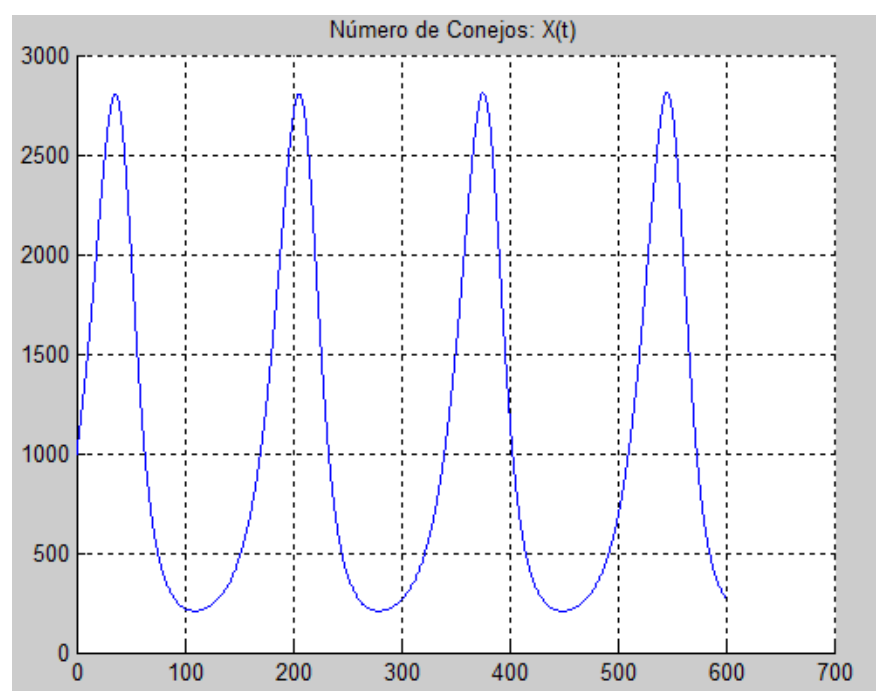

Fuente: Elaboración propia 


\section{Figura 6}

Comportamiento del número de predadores en el tiempo

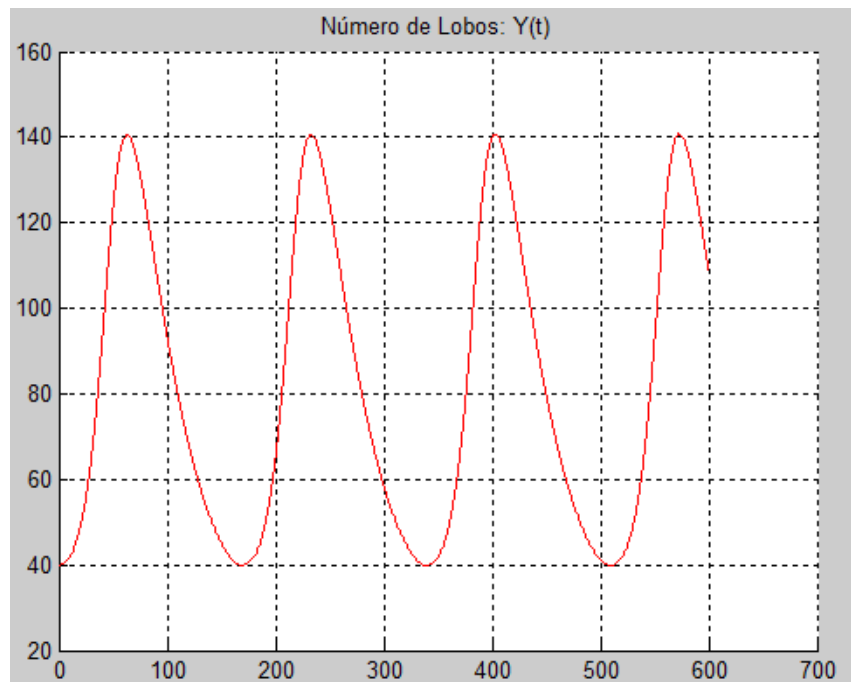

Fuente: Elaboración propia

\section{CONCLUSIONES}

Se aplicó y se analizó mediante un modelo matemático el modelo depredador - presa de Lotka Volterra, donde una población interactúa con poblaciones de otras especies. Donde además se considera que el grado de interacción es proporcional al número de individuos de cada especie.

\section{REFERENCIAS}

Figuereiro, A. (2014). Modelalagem de um sistema presa predador. Brasil. Universidade Federal do AMAPÁ.

López, J. (2012). Sistemas de Ecuaciones diferenciales II. Recuperado de: http://www.ugr.es/ jllopez/Clase28.pdf

Puchiri, L. (2018). Modelos de población en Ecuaciones diferenciales. PUC del Perú. Perú. 